\title{
APORTES METODOLÓGICOS DE UNA EXPERIENCIA DE CONSTRUCCIÓN DE INDICADORES DE DESARROLLO MEDIÁTICO
}

\author{
METHODOLOGICAL CONTRIBUTIONS OF AN EXPERIENCE \\ IN THE CONSTRUCTION OF MEDIA DEVELOPMENT INDICATORS
}

\section{Lidieth Garro-Rojas}

Universidad de Costa Rica, Costa Rica

(iD) https://orcid.org/0000-0003-3822-8468

\section{Lilliana Solís Solís}

Universidad de Costa Rica, Costa Rica

(iD https://orcid.org/0000-0001-7879-4910

\author{
Allan Monge Cordero \\ Universidad de Costa Rica, Costa Rica \\ (iD https://orcid.org/0000-0002-4642-8075
}

Autor para correspondencia: Lidieth Garro Rojas, email: lidieth.garro@ucr.ac.cr

\section{Resumen}

Este artículo da cuenta del proceso de reflexión crítica y definición metodológica para la creación de indicadores de desarrollo mediático, a partir de la Organización de las Naciones Unidas para la Educación, la Ciencia y la Cultura (UNESCO). Detalla las etapas que se siguieron en la definición de la propuesta, las cuales consistieron en la revisión de los indicadores y subindicadores propuestos por la UNESCO; la definición de un marco teórico y conceptual que orientara la creación de una propuesta ajustada a Costa Rica; la adecuación de los indicadores al concepto de ciudadanía comunicativa; y finalmente, la validación de los indicadores mediante el método Delphi. Esta reflexión teórica y metodológica sobre el proceso de adaptación de indicadores mediáticos a los contextos nacionales aporta al conocimiento de los medios de comunicación y al ejercicio de la ciudadanía comunicativa. El texto explica los pasos seguidos desde la adopción de la perspectiva de la ciudadanía comunicativa y el concepto de la complejidad, para crear una propuesta adaptada a la realidad nacional, la cual, si se aplica con la rigurosidad planteada, generará datos confiables sobre la estructura y funcionamiento de los medios de comunicación. En su conjunto el artículo responde a la pregunta: ¿cómo en Costa Rica se crearon y validaron indicadores de desarrollo mediático a partir de la propuesta de UNESCO?

Palabras clave: comunicación, indicadores, democracia, medios de comunicación, ciudadanía, metodología. 


\begin{abstract}
This article exposes the critical reflection process and methodological definition for the creation of media development indicators based on the United Nations Educational, Scientific and Cultural Organization (UNESCO). Besides, it details the stages that were followed to define the proposal. This one consisted of the review of indicators and sub-indicators proposed by UNESCO, the definition of a theoretical and conceptual framework that will guide the creation of a proposal adjusted to Costa Rica, the adaptation of the indicators to the concept of communicative citizenship and the indicator validation through the Delphi method. This theoretical and methodological reflection about the process of adapting media indicators to national contexts contributes to media knowledge and to the exercise of communicative citizenship. The authors explain the steps taken, from the adoption of the communicative citizenship perspective to the complexity concept, in order to create a proposal adapted to the national reality. If it is applied with the rigor recommended, it will generate reliable data about media structure and its performance. In fact, the article answers the following question: how were media development indicators created and validated in Costa Rica based on the UNESCO proposal?
\end{abstract}

Key words: communication, indicators, democracy, media, citizenship, methodology.

Recibido: 11/11/2020

Aceptado: 23/03/2021

\section{Introducción}

La elaboración de los indicadores ${ }^{1}$ de la UNESCO (2008) es el resultado de una amplia consulta internacional, que se desarrolló entre los años 2006 y 2008. Tal y como lo indica la resolución 1636 de la UNESCO la medición del desarrollo mediático es una herramienta de diagnóstico que permite la evaluación del nivel de desarrollo de los medios de comunicación en cada país, para de esa forma, determinar las acciones y políticas públicas que

\footnotetext{
${ }^{1}$ En el entorno de esta investigación, se entiende que un indicador es un dato que pretende reflejar el estado de una situación, o de algún aspecto particular, en un momento y
}

contribuyan a la gobernabilidad y el desarrollo democrático.

En su conjunto, los indicadores de la UNESCO proporcionan una guía para contar con un amplio mapa de la ecología mediática y conocer el estado de la libertad de expresión, el pluralismo y la diversidad de los medios de comunicación. Esta propuesta introduce la importancia de la medición y faculta establecer comparaciones. Sin embargo, se trata de una herramienta creada para aplicarse de manera generalizada y sin considerar los diferentes contextos nacionales, al igual que los desafíos

un espacio determinados. El sistema de indicadores del que da cuenta este artículo pretende reflejar el grado en que el sistema nacional de medios favorece el ejercicio de la ciudadanía comunicativa.

Global Media Journal México, 18(34), 1-17, enero - junio 2021. 
específicos en materia de medios de comunicación.

Por ejemplo, en el caso de Costa Rica, el país exhibe una amplia legislación sobre regulaciones a la libertad de expresión comercial, pero es ayuno en normativa y política pública para promover procesos de comunicación más democráticos, en especial para garantizar el acceso de sectores sociales al espectro radioeléctrico y a los contenidos audiovisuales. En ese sentido, la creación de indicadores nacionales posibilitaría que el país mida la calidad de su democracia comunicativa.

Además, para que los indicadores de la UNESCO potencien, en cada entorno nacional, su utilidad como herramientas para fomentar la democracia comunicativa, deben pasar por procesos de revisión crítica y adaptación. Este artículo aborda el proceso metodológico seguido en Costa Rica para adaptar la propuesta de la UNESCO al entorno mediático local, que se inició en el año 2016. Reflexiona y da cuenta del proceso de análisis de la propuesta base y la creación de los indicadores nacionales partiendo del replanteamiento conceptual hasta la validación por parte de especialistas. Detalla las cuatro etapas que se siguieron en la definición de la propuesta de indicadores para Costa Rica: a) la revisión de los indicadores y subindicadores de la UNESCO; b) la definición de un marco teóricoconceptual que oriente la creación de una propuesta ajustada a Costa Rica; c) la adecuación de los indicadores al concepto de ciudadanía comunicativa $\mathrm{y}$, finalmente, d) la validación de los indicadores mediante el método Delphi.

La adaptación de indicadores mediáticos a los contextos nacionales permite generar datos confiables sobre la estructura y el funcionamiento de los medios de comunicación, además de contar con una línea base para estudios futuros y para la toma de decisiones sobre políticas públicas en relación con medios de comunicación.

\section{Abordaje teórico-metodológico}

Se inició el análisis detallado y se procedió a la creación de un marco conceptual y metodológico, con el fin de adaptar la propuesta a la realidad mediática costarricense. La orientación teórica de todo este proceso se estableció desde los conceptos de ciudadanía comunicativa y complejidad, los cuales se discuten a continuación.

\section{Ciudadanía comunicativa: eje orientador de la propuesta}

La sociedad costarricense posee un amplio acceso a las tecnologías de la información y la comunicación; la vida social y los medios de comunicación, juegan un papel central en la escenificación de la vida política y social. En ellos se difunden, replican y debaten las ideas, discursos y proyectos en torno al modelo de sociedad, por lo cual el ejercicio de la ciudadanía es insoslayablemente una práctica comunicativa que se desarrolla en un espacio público mediatizado. De ahí la trascendencia de conocer el funcionamiento del sistema de medios para evaluar las condiciones que favorecen la práctica de la ciudadanía.

Global Media Journal México, 18(34), 1-17, enero - junio 2021. 
Como punto de partida del desarrollo teórico conceptual, se establece la ciudadanía comunicativa como eje de la propuesta y se define que el propósito de los indicadores debe ser evaluar el desarrollo mediático costarricense desde esta perspectiva, lo que supone dar cuenta de las condiciones para el ejercicio democrático en un entorno altamente mediatizado.

Si bien en el ámbito del ejercicio de la democracia los medios de comunicación están entre las instituciones más importantes, Virilio (1996) afirma que el "cuarto poder" es "la única de nuestras instituciones capaz de funcionar al margen de todo control democrático eficaz, ya que cualquier crítica independiente dirigida contra él, cualquier solución de recambio, son desconocidas por el gran público, simplemente porque no tienen ninguna posibilidad de ser difundidas con amplitud" (p. 11).

Es decir, los medios de comunicación se construyen sobre una paradoja: mientras es ampliamente generalizado el rechazo de todo tipo de censura, ellos definen qué difundir y cómo hacerlo, sin tener que dar cuenta de sus procesos de toma de decisión, vinculaciones empresariales o institucionales frente a sus audiencias (Virilio, 1996). En las sociedades mediatizadas esto confronta una "legitimidad informacional con un poder distinto al de la legitimidad democrática" (Mata, 2006, p. 7).

En este sentido, es útil la noción de ciudadanía comunicativa, pues, al partir del "reconocimiento de la capacidad de ser sujeto de derecho y demanda en el terreno de la comunicación pública" (Mata, 2006, p. 13), se coloca la mirada del análisis en las condiciones existentes para el uso de los medios de comunicación, en tanto plataforma para el ejercicio de esos derechos (Córdoba, 2008; Mata, 2006).

El ejercicio de la ciudadanía comunicativa apunta a generar las condiciones para alcanzar una sociedad de verdaderas personas ciudadanas y remite también, a la capacidad de agencia de los sujetos en su expresión comunicativa; retoma las consideraciones republicanas sobre el "compromiso de las personas con la "cosa pública", mediante "la participación en la deliberación pública y en la contingencia política, y el compromiso activo en la construcción colectiva de proyectos de sociedad" (Hopenhayn, 2005, p. 215, citado por Mata, 2006).

Para conocer el aporte específico de los medios de comunicación y otros actores que conforman el entorno mediático para el ejercicio de la ciudadanía comunicativa, se desarrolló un modelo de evaluación basado en cuatro conceptos orientadores:

Libertad de expresión: se refiere al derecho de investigar, buscar, producir, difundir y recibir información, opinión e ideas, sin censura previa, a través de los medios de comunicación y de cualquier otro procedimiento, en el marco del respeto al Estado de derecho democrático. Se establece en la Constitución Política y tratados internacionales signados por el Gobierno costarricense.

Pluralidad: según Lovatón (2014), el concepto de pluralidad implica una doble condición; por un lado, la pluralidad interna de los medios de comunicación que refiere al derecho que asiste a los periodistas a expresarse y a difundir sus ideas e información en forma independiente, libre y plural, 
dentro de su espacio laboral; y por otro lado, la pluralidad externa, que alude a la necesidad de las sociedades democráticas de contar con medios de comunicación que expresen puntos de vista distintos y discrepantes, capaces de representar los puntos de vista de diversos actores sociales.

Diversidad: se refiere a que los medios de comunicación, en su conjunto, como sistema mediático, representen en sus contenidos todo el espectro de intereses y visiones políticas como sociales. Procura la maximización del número y la diversidad de voces que pueden participar en la deliberación pública y en los procesos democráticos, a través de los medios de comunicación de distinta naturaleza: públicos, comerciales y comunitarios.

Pluralismo y diversidad son condiciones necesarias para el ejercicio de un debate ciudadano amplio; la concentración de los medios en pocas empresas implicaría la falta de pluralidad externa, lo cual supone un daño potencial a la democracia y a las condiciones para el ejercicio de la ciudadanía comunicativa. La diversidad posibilita una polifonía de voces, aporta a la calidad de una democracia en la que los sujetos no son únicamente portadores de derechos (pasivos) sino que pueden acceder a los medios de comunicación para profundizar el ejercicio y demandar nuevos derechos.

Acceso: remite a de las condiciones necesarias para que la población en general acceda a

\footnotetext{
${ }^{2}$ Mondragón (2002), citando fuentes de la ONU, señala que los indicadores son: "herramientas para clarificar y definir, de forma más precisa, objetivos e impactos (...) son medidas verificables de cambio o resultado (...) diseñadas para contar con un estándar contra el cual evaluar, estimar o demostrar el progreso (...) con respecto
}

las diversas plataformas mediáticas, tanto en términos de la existencia de políticas públicas, tecnologías, capacitación para su uso, disminución de las brechas, participación real, lenguajes y particularidades culturales e, incluso, propiedad.

La evaluación de las condiciones del desarrollo mediático, mediante la creación de un conjunto de indicadores ${ }^{2}$, sólo es posible a partir de un sistema de los mismos que considere temáticas, aspectos y poblaciones muy diversas vinculadas al uso y la gestión de los medios de comunicación. Para la creación de ese abordaje se requiere no solamente recurrir a diferentes aportes conceptuales provenientes de distintos campos disciplinares, sino a diversas técnicas de recolección de información.

\section{La complejidad: marco para la construcción} de indicadores de desarrollo mediático

La multidimensionalidad del problema de crear indicadores para evaluar el desarrollo mediático se realiza desde el concepto de complejidad.

La evaluación del ejercicio de la ciudadanía comunicativa $^{3}$, como otros temas y problemas complejos, requiere un sistema diverso y amplio de indicadores, para cuya elaboración es necesario recurrir a diferentes particularidades conceptuales.

Por ello, la perspectiva metodológica que se asume es la de la complejidad, que parte de la

a metas establecidas, facilitan el reparto de insumos, produciendo (...) productos y alcanzando objetivos" (p. 52).

${ }^{3}$ Posteriormente se explica la elección de ese abordaje conceptual y se desarrollan sus aspectos principales. 
construcción de conocimiento transdisciplinar. Como expone Paredes (2007), "la transdisciplinariedad no posee como las disciplinas un método y un objeto de estudio específico, tampoco estudia un solo nivel de realidad o un segmento de dicho nivel, sino que se dedica al estudio de la dinámica que se genera por la actuación de diversos niveles de realidad y percepciones al unísono" (p. 38).

Con Morin (2010), se reconoce que existen diferentes niveles y dimensiones de la realidad; que interesa su comprensión multidimensional, sin pretensiones de llegar a conclusiones definitivas y que se abre a diversas realidades subjetivas como una forma de proponer el análisis.

En el proceso de reformulación de los indicadores confluyen conocimientos y experiencias que provienen de campos como la comunicación, el derecho, la sociología y la estadística. Ello corresponde un enfoque que procura la creación de conocimiento orientado por problemáticas sociales y no determinado en forma exclusiva por fronteras disciplinarias previamente establecidas.

La reflexión en torno a los conceptos de complejidad y ciudadanía comunicativa, y su aplicación, condujo a la reelaboración de las categorías, indicadores y subindicadores que se detalla en la Etapa 2: "Adecuación de los indicadores al concepto de ciudadanía comunicativa".

\section{Etapas en la construcción y validación de la propuesta de creación del sistema de indicadores para Costa Rica}

\section{a) Etapa 1: Resultados de la revisión de los indicadores propuestos por la UNESCO}

La primera etapa consistió en la minuciosa revisión de la propuesta de la UNESCO y el análisis de su aplicación en distintos países latinoamericanos. Ello permitió identificar debilidades en el documento. Entre las más importantes:

- Reiteraciones de indicadores y subindicadores.

- Algunas inconsistencias internas del documento, en términos de la articulación entre categorías y subcategorías, subcategorías e indicadores e indicadores y subindicadores.

- Fundamentación teórica insuficiente, con falta de definiciones conceptuales y operativas para enmarcar y dar coherencia a los indicadores y subindicadores.

- Existencia de indicadores y subindicadores construidos a la luz de otros contextos e inadecuados para su aplicación en Costa Rica.

- Carencia de perspectiva de género en muchos de los indicadores y subindicadores.

En esta etapa del proceso se realizó un espacio de consulta con personas investigadoras de la comunicación del ámbito académico, quienes 
coincidieron con la mayoría de las inquietudes del equipo de investigación, consulta que validó la decisión de reelaborar integralmente el documento, a partir de la definición de un marco teóricoconceptual.

\section{b) Etapa 2: Adecuación de los indicadores al concepto de ciudadanía comunicativa}

Con base en la definición de la noción de la ciudadanía como eje orientador y del reconocimiento de la complejidad como perspectiva metodológica válida, se inicia la revisión crítica y actualización de la totalidad de los indicadores. Conforme a la noción de complejidad, se reconoce la existencia de distintos niveles y perspectivas para su abordaje.

La perspectiva se refiere al punto de vista de variados sujetos: el Estado, los medios de comunicación, los individuos y las instituciones internacionales.

Los niveles aportan a la comprensión de los diferentes espacios o escenarios en los que se realiza el análisis mediático: en la normativa, en la gestión institucional, la operación de los propios medios de comunicación y en la actuación ciudadana.

En la interacción de las diferentes perspectivas y niveles se concretan indicadores referidos a:

- la normativa sobre los medios de comunicación;

- las políticas públicas sobre la comunicación que tienen una incidencia directa en el ejercicio de la democracia;

- las opiniones, valoraciones y prácticas institucionales de los propios medios de comunicación sobre las condiciones institucionales para su trabajo;

- las opiniones, compromisos y prácticas de la sociedad civil en torno al sistema mediático;

- las opiniones de la ciudadanía sobre las condiciones para la participación en los medios de comunicación y la valoración de sus propias prácticas;

- la valoración de los profesionales de la comunicación sobre las condiciones y sus prácticas para el ejercicio profesional; $\mathrm{y}$

- la valoración relacionada con los aportes de los contenidos de la educación superior sobre aspectos que contribuyen a fortalecer la perspectiva democrática y de derechos entre las personas profesionales de la comunicación.

La Imagen 1 da cuenta de la complejidad del ejercicio de reflexión desarrollado.

La nueva estructura modifica, de manera significativa, la propuesta de la UNESCO, al pasar de cinco categorías a seis, así como se reestructuran todos los temas. Además, se rehacen y proponen nuevos indicadores y subindicadores.

La propuesta final consta de seis categorías:

- El sistema regulador de los medios de comunicación favorece la libertad de expresión, el pluralismo y la diversidad mediática.

- Las políticas y el accionar del Estado garantizan un sistema de medios plural y diverso. 
- Los medios sirven como plataforma efectiva para el ejercicio democrático.

- Los medios cuentan con instrumentos de gestión y desarrollan prácticas democráticas.

- Las condiciones laborales del personal mediático permiten un ejercicio profesional libre y seguro.

- La oferta formativa para profesionales de la comunicación favorece la libertad de expresión, el pluralismo y la diversidad.
Seguidamente se procede a validar los indicadores en varios sentidos. En primer lugar, se realiza una revisión de la coherencia interna; se analiza la articulación lógica existente entre los distintos elementos de la propuesta: categorías, temas, indicadores y subindicadores.

En un segundo momento se revisan los elementos, en atención a la coherencia teórica y la interna; se eliminan subindicadores, otros se replantean y se agregan nuevos aspectos por evaluar.

\section{Imagen 1}

Propuesta metodológica: categorías, perspectivas y niveles de análisis

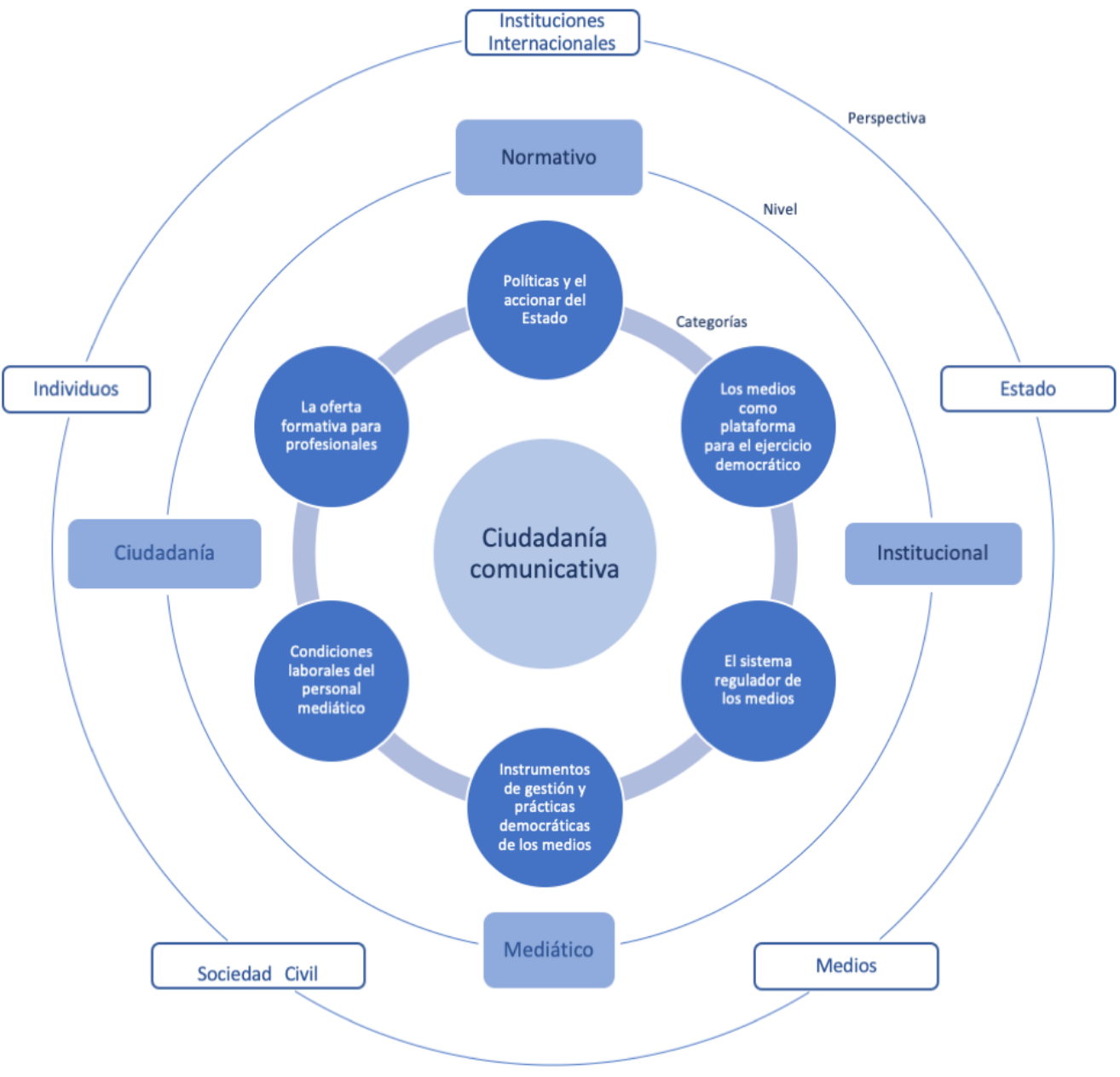

Global Media Journal México, 18(34), 1-17, enero - junio 2021. 
Se contó con el apoyo de personas expertas para precisar aspectos puntuales en materia de género, tecnologías, acceso, seguridad periodística y normativa. La etapa concluye con la decisión de someter la propuesta a una validación externa, la cual permita incorporar el criterio de personas profesionales de la comunicación, las leyes, los derechos humanos e, igualmente, personas ligadas al mundo de la política, actores sociales, empresas de comunicación y organizaciones gremiales.

\section{c) Etapa 3: Validación de indicadores mediante el método Delphi}

La validación externa ${ }^{4}$ se realiza mediante la aplicación del método Delphi, el cual posibilita obtener la opinión colectiva de un grupo diverso de actores interesados en los medios de comunicación, en coherencia con la búsqueda de diferentes perspectivas sobre el tema estudiado.

Se validan las categorías, los temas y los indicadores y, para la etapa final, se deja el ajuste de los subindicadores, debido a que el número sobrepasaba los 200 y hace inviable la utilización de la técnica.

Además de conocer e incorporar la opinión del grupo de personas expertas con respecto a la propuesta, esta etapa explora las condiciones que legitimen su aplicación y su uso como un

\footnotetext{
4 Se agradece a los investigadores del Centro de Investigación y Capacitación en Administración Pública (CICAP), que desarrollan el Índice Costarricense de Transparencia Institucional, quienes compartieron su experiencia para orientar el proceso de validación utilizando la metodología Delphi.

5 Distinta literatura revisada se refiere al Delphi como
}

instrumento para conocer el estado de desarrollo de los medios, políticas públicas en comunicación y derechos humanos.

\section{Descripción del método Delphi}

El llamado método Delphi ${ }^{5}$ es un proceso mediante el cual un grupo de expertos es consultado reiterativamente al menos en dos ocasiones sobre el mismo asunto, de forma tal que las personas participantes pueden reconsiderar su respuesta con base en la información que reciben del resto de expertos que forman el panel. En la literatura académica en castellano es ampliamente reconocida su utilidad para la construcción de indicadores (De Castro y López Padrón, 2013, 2014; MonagasDocasal, 2013; Trapote, 2015), la toma de decisiones y el de orientar decisiones en el futuro (Aceituno, 2013; Torres et al., 2016).

Consiste en la aplicación repetida de rondas de preguntas que faciliten depurar el juicio grupal, el cual se obtiene mediante procedimientos matemáticos de agregación de juicios individuales. Luna et al. (2005) refieren que en cada nueva vuelta se informa a los panelistas sobre la concentración y la dispersión de las respuestas en la fase anterior, se les recuerda cuál fue la posición que cada uno de ellos o ellas adoptó y se les pide que la corroboren o rectifiquen.

método o como técnica. Luna et al. (2005) lo denominan indistintamente como un método o una técnica mientras que García-Aracil y Palomares-Montero (2012) lo refieren como un método. Debido a que involucra una diversidad de pasos que incluyen técnicas de carácter cualitativo y cuantitativo, preferimos la nominación más extendida de Método Delphi. 
La pretensión es obtener una opinión grupal, construida a partir del criterio de expertos. Ésta, según Luna et al. (2005), se apoya en tres pilares fundamentales: 1) el mantenimiento del anonimato de los participantes; 2) la retroalimentación controlada; y 3) la respuesta estadística representativa del grupo.

Se trata de un método que aprovecha el conocimiento de personas expertas en el que se han eliminado las interacciones sociales dentro del grupo, controlando, de este modo, aspectos como las influencias que se derivan de las jerarquías formales y simbólicas (Aceituno, 2013; Silva et al., 2002). Las respuestas son anónimas en cada ronda, con lo cual se desconoce cómo votan las demás personas, excepto en la forma de agregado estadístico.

Luna et al. (2005) detallan que en la base del método Delphi existe el criterio de la superioridad de un juicio grupal sobre el juicio individual; los juicios individuales son insuficientes pues se trata de resolver problemas complejos, como el investigado, en condiciones de incertidumbre y cuando existe escasa información disponible. A diferencia de otras técnicas cualitativas, como los grupos focales o las entrevistas grupales, permite obtener información y opiniones de sujetos físicamente alejados y posibilita la generación de ideas con respuestas abiertas, de forma bien estructurada y con un componente cualitativo añadido.

Individualmente, las personas expertas participan en forma anónima y a título personal, y no como representantes de instituciones $u$ organizaciones con las cuales tienen vinculación. El cambio de opinión y la defensa de los argumentos se hace sin una interacción directa con el grupo. Las personas organizadoras realizan una devolución controlada, en el que la opinión de todos los participantes se presenta de forma estadística, con indicación del grado de consenso que se ha obtenido.

Además, Luna et al. (2005) sugieren las siguientes condiciones en las que la aplicación del método Delphi es adecuado cuando:

- no existe información disponible, por lo que es necesario recurrir al juicio de personas expertas;

- el asunto por tratar no se presta para el uso de una técnica analítica precisa, pero sí puede beneficiarse de juicios subjetivos sobre bases colectivas;

- se necesita o es conveniente la participación de más personas expertas de las que pueden interactuar cara a cara en un encuentro fructífero;

- por los costos que implica no es posible realizar una gran cantidad de encuentros presenciales de grupo;

- se procura la heterogeneidad de las personas participantes, por lo que es preferible este método a los encuentros cara a cara. Se favorece así la participación de personas con diferentes formaciones y ubicación geográfica; $y$,

- se controla el peso que en la interacción social tienen la autoridad o el prestigio en los encuentros cara a cara.

Dos elementos fundamentales del método Delphi son: el panel de expertos y el cuestionario. Sobre el panel de expertos, Luna et al. (2005) exponen que la 
selección debe recaer sobre las personas más competentes en la materia; de ser posible, debe buscarse la conformación con miembros de sectores y grupos diversos; el tamaño del grupo puede oscilar entre 15 y 25 personas si se trata de un grupo heterogéneo, y entre 10 y 15 en el caso de grupos homogéneos. Aceituno (2013) señala que desde distintas experiencias se hacen diversas recomendaciones para que la aplicación de la técnica se considere válida y óptima y oscila en un rango no inferior a siete ni mayor a 30 .

Una de las limitaciones se deriva precisamente de la conformación del panel de expertos, pues en su composición debe procurarse que, además del conocimiento o la experiencia específica, se trate de participantes con compromiso en el proceso, con habilidades de comunicación escrita y con el tiempo y la disponibilidad suficientes. Según García-Aracil y Palomares-Montero (2012) se reportan elevadas tasas de abandono en su aplicación; asimismo, se indica la incertidumbre que se deriva de no poder controlar directamente la forma en la que se completan los cuestionarios.

En cuanto al cuestionario, se aplican las indicaciones comunes sobre su construcción, tales como concreción y precisión, así como una cantidad de preguntas manejable, además de que el tiempo de respuesta no sobrepase los 30 minutos.

El proceso de selección del panel de expertos, siguiendo las recomendaciones señaladas en Luna et al. (2005), recayó en personas que, por sus calidades académicas y experiencia profesional, representan sectores y grupos diversos, vinculados con la comunicación y el sistema de medios costarricense. El número de expertos necesarios para realizar el estudio se sustentó en las consideraciones dadas por Luna et al. (2005).

\section{Selección del panel y aplicación del cuestionario}

Los criterios de conformación del panel partieron de la intención de contar con la representación de diversos sectores y actores sociales. Para ello se tomaron en consideración las calidades académicas, la experiencia profesional, la paridad de género, la representación de sectores y grupos diversos, relacionados con la comunicación y el sistema de medios costarricense. También, la disposición manifestada durante un primer acercamiento por vía telefónica, en el que se les explicó a las personas invitadas los alcances de la investigación y se les solicitó integrar el panel.

Se identificaron 42 personas a las que se les explicó la investigación y se les solicitó colaborar: ocho del sector académico; cinco del profesional de la comunicación; 12 del empresarial; ocho personas de la sociedad civil; cuatro representantes del sector político y cinco profesionales en derecho. De estos, 30 estuvieron anuentes a participar de la validación y se les envió el instrumento. Al final, respondieron 19 personas todo el proceso: nueve mujeres y 10 hombres.

En cuanto a las personas participantes, estas tienen formación en comunicación, derecho, sociología y negocios, con grado académico de doctorado (4), maestría (6) y licenciatura (7), y dos personas sin grado conocido. 
Antes de enviar la consulta al panel de expertos, se procedió a efectuar una prueba del instrumento con una persona experta, quien proporcionó valiosas recomendaciones y sugerencias. La carpeta que recibió cada uno de los participantes contenía, además de una nota explicativa con los objetivos del estudio y lo que se esperaba de cada experto, los siguientes documentos:

1) Instructivo; un archivo conformado por varias hojas de trabajo en las que se podía ver las Instrucciones. Ronda 1; Estructura propuesta; Resumen de indicadores; Cuestionario de categoría 1 a la 6 .

2) Consentimiento informado; este es un documento que detalla los alcances de la investigación, el carácter anónimo de las respuestas y los productos esperados.

3) El listado de categorías e indicadores, el cual incluye las seis categorías que, a su vez, se dividen en temas, de los que se derivan indicadores y subindicadores.

El instrumento solicitaba al panel de expertos la evaluación de cada uno de los indicadores de manera individual. La única respuesta obligatoria consistía en aceptar o rechazar cada subindicador, con un "sí" o un "no". Adicionalmente, la persona consultada podía incluir una propuesta de mejora de la descripción y, en caso de responder negativamente a la inclusión, se le solicitaba fundamentar esa razón.

La aplicación se realizó en el mes de mayo de 2019. Al concluir la fecha límite señalada por el equipo de investigadores para la primera ronda de valoración, se recibieron 19 respuestas, con niveles variados de observaciones, las cuales fueron analizadas y ponderadas, según el proceso que establece el método.

En relación con la validación de los ítems que efectúa el panel de personas expertas, Silva et al. (2002) establecen cuatro niveles, de acuerdo con:

- Mayoría: ocurre cuando más del 50\% de los participantes exhibe consistencia en la respuesta. En este caso se acepta el ítem evaluado.

- Bipolaridad: cuando las personas participantes están igualmente divididas sobre alguna pregunta. Con base en las respuestas cualitativas, se puede eliminar o reescribir. En este segundo caso se lleva a cabo una nueva ronda para cerciorarse de que pasa la prueba de la estabilidad o si debe ser retirada.

- Pluralidad: sucede cuando una gran parte del grupo, pero menos del 50\%, alcanzan acuerdo. En este caso se revisa el consenso dentro del grupo y la naturaleza del acuerdo, con el fin de buscar estabilidad o reescribir el ítem.

- Desacuerdo: se presenta cuando cada participante mantiene puntos de vista independientes de los demás, de manera que no puede obtenerse consonancia en las respuestas. Cuando hay un desacuerdo estable, se debe tomar la decisión de eliminar el ítem o reescribirlo y someterlo, nuevamente a la consideración del grupo.

Las respuestas se analizan estadísticamente, para lo cual se utilizan la mediana y la dispersión, 
sintetizando al respecto respuestas que son presentadas al grupo en rondas posteriores (Silva et al., 2002).

Al finalizar el ejercicio con las personas expertas, se promediaron las respuestas para cada indicador y se aceptaron los indicadores que obtuvieron un promedio de aceptación superior al 50 por ciento. Además, se analizaron las observaciones incluidas en la columna de "mejora la descripción" y se procedió a escribir cuando correspondía, un nuevo indicador o a mejorar la redacción. Las respuestas obtenidas incluyeron precisiones conceptuales, señalamientos de posibles duplicaciones, peticiones de eliminación, con base en las interpretaciones conceptuales y solicitudes de incluir nuevos indicadores.

El resultado del proceso anterior se consolidó en un documento que se reenvió a los expertos para que hicieran un segundo ejercicio de análisis. En esta segunda ronda de validación se recibieron solamente dos observaciones, por lo que se procedió a dar por concluido el ejercicio y a redactar la versión final de indicadores para medir el desarrollo mediático costarricense.

\section{d) Etapa 4: Propuesta final ajustada}

La propuesta sometida a dos rondas de validación mediante la utilización del método Delphi se consolida en un documento, en el cual se incluyeron las modificaciones de redacción y descripción, tanto en lo relacionado con los temas como con los indicadores.

El resultado de la primera ronda, como producto de las sugerencias de mejora, modificó una de las categorías; se ajustó la redacción de tres temas y la descripción de 13. En lo referido a los indicadores, se produjo un ajuste en la redacción de 12 y en la descripción de 44 indicadores, además, se eliminaron tres indicadores y se incorporó uno nuevo.

Cabe destacar que ninguno de los indicadores fue eliminado en razón de una baja calificación, sino como producto de ajustes conceptuales o reiteraciones. El nuevo indicador incluido responde a la necesidad de puntualizar el respeto a la legislación en torno a la seguridad social en Costa Rica, en lo relativo al personal mediático. El resultado final se resume en la Tabla 1.

La propuesta para analizar el desarrollo mediático, en clave de ciudadanía comunicativa, queda constituida por seis categorías, 25 temas, 65 indicadores y 223 subindicadores. Luego de la validación Delphi, como ya se indicó, el equipo de investigación ajustó los subindicadores a la descripción final de cada uno de los indicadores.

El modelo de evaluación se completa con una serie de 22 instrumentos de recolección de información, formulados conforme a la última versión de indicadores y subindicadores. 


\section{Tabla 1}

Indicadores de desarrollo mediático según temas, indicadores y subindicadores por categoría

\begin{tabular}{lccc}
\hline \multicolumn{1}{c}{ Categoría } & Temas & Indicadores & Subindicadores \\
\hline $\begin{array}{l}\text { El sistema regulador de los medios de comunicación } \\
\text { favorece la libertad de expresión, el pluralismo y la } \\
\text { diversidad mediática. }\end{array}$ & 3 & 15 & 65 \\
$\begin{array}{l}\text { Las políticas y el accionar del Estado garantizan un } \\
\text { sistema de medios plural y diverso. }\end{array}$ & 6 & 13 & 46 \\
$\begin{array}{l}\text { Los medios sirven como plataforma efectiva para el } \\
\text { ejercicio democrático. }\end{array}$ & 5 & 10 & 33 \\
$\begin{array}{l}\text { Los medios cuentan con instrumentos de gestión y } \\
\text { desarrollan prácticas democráticas. }\end{array}$ & 3 & 10 & 30 \\
$\begin{array}{l}\text { Las condiciones laborales del personal mediático } \\
\text { permiten un ejercicio profesional libre y seguro. }\end{array}$ & 5 & 11 & 32 \\
$\begin{array}{l}\text { La oferta formativa para profesionales de la } \\
\text { comunicación favorece la libertad de expresión, el } \\
\text { pluralismo y la diversidad. }\end{array}$ & 3 & 6 & 17 \\
\begin{tabular}{l} 
Total \\
\hline
\end{tabular} & 25 & 65 & 223 \\
\hline
\end{tabular}

\section{Conclusiones}

La creación de una propuesta para medir el desarrollo mediático costarricense se hace desde una perspectiva contextualizada en América Latina, y en particular de Costa Rica, país en el que los medios de comunicación ocupan un lugar central en el ejercicio democrático.

El proceso, que dio inicio con la revisión en profundidad y adecuación de la propuesta de la UNESCO, desde la profundización en el concepto de ciudadanía comunicativa, aporta al propósito general de contar con un modelo evaluación que permita conocer tanto el desarrollo como el aporte de los medios de comunicación a la democracia, a partir de la aplicación de una metodología de evaluación que provea información relevante para una discusión informada.

Elaborados desde una perspectiva de ciudadanía comunicativa, se procura que los indicadores faculten responder a la pregunta sobre las condiciones de funcionamiento de los medios de comunicación. La información obtenida, una vez aplicados estos, permitirá reconocer qué aspectos de los medios se pueden mejorar, visibilizando al respecto las carencias y las fortalezas.

El concepto de ciudadanía comunicativa enriquece los indicadores mediáticos, pues permite comprender la doble dimensión de los medios de 
comunicación: como un derecho ciudadano en sí mismos y una plataforma que brinda a la ciudadanía la posibilidad de participar en las discusiones públicas para ampliar y fortalecer otros derechos. Desde esta perspectiva, las personas acceden a los medios de comunicación, en tanto espacios de vivencia democrática y ejercicio de derechos. El concepto se fortalece al introducir en la propuesta de evaluación cuatro principios orientadores que deben caracterizar una sociedad democrática: libertad de expresión, diversidad, pluralidad y acceso.

El abordaje desde una visión latinoamericana del concepto canaliza la discusión para contar con medios de comunicación fuertes frente a los Estados, libres e independientes, con sentido de responsabilidad, con espacios de participación abiertos a la ciudadanía que garanticen un entorno mediático plural y diverso. Una ciudadanía que conoce sus responsabilidades y derechos frente a los medios de comunicación, que posee conocimientos y habilidades para comprenderlos y hacer demandas, según sus propias agendas y participar en ellas. Los medios se robustecen al contar con profesionales formados en un sistema educativo de calidad, con conocimiento y criterio para reconocer el entorno democrático y fortalecer la perspectiva de derechos, con prácticas éticas, que, del mismo modo, trabajan en condiciones seguras y apegadas al marco legal.

La elaboración de la propuesta se hace desde el abordaje de la complejidad, de manera que se articulan diversas perspectivas de actores, distintos niveles de análisis, al igual que categorías conceptuales y disciplinas. La propuesta se enriqueció con el aporte de expertos de la academia y personas con reconocida experiencia, quienes se desenvuelven en el ámbito laboral, así como de la sociedad civil y el campo político, además de la incorporación de diferentes perspectivas, entre ellas, la normativa, las políticas públicas, laborales, culturales, de seguridad, entre otras.

El sistema de indicadores propuesto, teórico y metodológicamente sólido posibilita crear una línea base de análisis y ser, además, sostenible en el tiempo.

El uso del método Delphi permitió una conjunción de miradas y de actores sociales, que validaron la propuesta desde diferentes perspectivas disciplinarias, orientaciones políticas y experiencias personales, sin que el proceso se viera afectado por los sesgos individuales. Además, facilitó la realización de una consulta amplia, al igual que procesar los distintos aportes de una manera rigurosa.

La versión final de la propuesta de indicadores evalúa la calidad de nuestra democracia desde un enfoque innovador, que cuestiona la naturalización del aporte democrático de los medios de comunicación y demanda la producción de evidencia que constate la magnitud de esa contribución.

El ejercicio académico se completa con la creación de una batería de instrumentos de recolección de la información, los cuales responden a cada una las categorías y dimensiones que se ha considerado pertinente de analizar.

Así, el uso del modelo permitirá hacer valoraciones sobre la normativa vigente en el país, las políticas públicas efectivas, las prácticas éticas y 
de autorregulación en los medios de comunicación, la oferta y calidad de las formación y capacitación del personal mediático, el ejercicio profesional de la comunicación, entre otros.

La generación de datos confiables sobre la estructura y el funcionamiento de los medios de comunicación orientará sobre la formulación de políticas públicas, que coadyuven a una mayor democratización del entorno mediático, favorecedora del ejercicio de la ciudadanía comunicativa.

\section{Referencias bibliográficas}

Aceituno, O. P. (2013). Prospectiva y partidos políticos: escenarios para los próximos 15 años en Chile. Ril Editores.

Córdoba, M. L. (2008). Democracia comunicativa: nuevas formas para la intervención ciudadana. Anagrama, 6(12) 70-91. https://bit.ly/3eJb81I

De Castro, A. F., y López Padrón, C. A. (2013). Validación mediante método Delphi de un sistema de indicadores para prever, diseñar y medir el impacto sobre el desarrollo local de los proyectos de investigación en el sector agropecuario. Revista Ciencias Técnicas Agropecuarias, 22(3), 54-60. https://bit.ly/3y9mfJ5

De Castro, A. F., y López, A. (2014). Validación mediante criterio de usuarios del sistema de indicadores para prever, diseñar y medir el impacto en los proyectos de investigación del sector agropecuario. Revista Ciencias Técnicas Agropecuarias, 23(3), 77-82. https://bit.ly/3tIgbnt

García-Aracil, A. y Palomares-Montero, D. (2012) Indicadores para la evaluación de las instituciones universitarias: validación a través del método Delphi. Revista española de documentación científica. 35(1), 119-144. https://doi.org/10.3989/redc.2012.1.863

Hopenhayn, M. (2005). América Latina desigual y descentrada. Editorial Norma.

Luna, P., Infante, A. y Martínez, F. J. (2005). Los Delphi como fundamento metodológico predictivo para la investigación en sistemas de información y tecnologías de la información (IS/IT). Pixel-bit. Revista de Medios y Educación, (26), 89-112. https://bit.ly/2QfV6TP

Lovatón, D. (2014). El equilibrio interamericano entre pluralidad de información y concentración de medios. Derecho PUCP, (73), 131-153. https://doi.org/10.18800/derechopucp.201402.005

Mata, M. C. (2006). Comunicación y ciudadanía. Problemas teórico-políticos de su articulación. Fronteiras, estudos mediáticos, 8(1), 5-15. https://bit.ly/2QdxSxs

Monagas-Docasal, M. (2013). Procedimiento metodológico para la medición del capital intelectual en empresas hoteleras. Ingeniería Industrial, 34(1), 50-63. https://bit.ly/3w2SGqH

Global Media Journal México, 18(34), 1-17, enero - junio 2021. 
Mondragón Pérez, A. R. (2002). ¿Qué son los indicadores? Revista de Información y análisis, (19), 52-58. https://bit.ly/3yc2f8D

Morin, E. (2010). Sobre la interdisciplinariedad. Orientaciones Universitarias, (43), 9-17. https://bit.ly/3yaG8j6 Organización de las Naciones Unidas para la Educación, la Ciencia y la Cultura (2008). Indicadores de desarrollo mediático: marco para evaluar el desarrollo de los medios de comunicación social. UNESCO. https://bit.ly/2Qelcqa

Paredes, G. (2007). Críticas epistemológicas y metodológicas a la concepción positivista en las ciencias sociales. Academia, VI(12), 24-42. https://bit.ly/2SRGye9

Silva, S. A., Galindo, Y. y Mendoza, L. E. (2002). Nivel de satisfacción del usuario de las carreteras: método Delphi. Acta Universitaria, 12(3), 41-55. https://doi.org/10.15174/au.2002.280

Torres, M. C., Alarcón, J., Berhert, A., Cantero, V., Llanquipichún, D., Sáez, D. y Yáñez, I. (2016). Modelo de certificación de calidad para la gestión del cuidado en hospitales chilenos. Revista de Enfermagem Referência. IV(9), 65-74. https://doi.org/10.12707/RIV15015

Trapote, F. R. (2015). Procedimiento metodológico para evaluar el enfoque de género en los proyectos de desarrollo local. Editorial Universitaria.

Virilio, P. (1996). El arte del motor: aceleración y realidad virtual. Manantial. 